\title{
Corrigendum to "Pharmacological Basis for Use of Selaginella moellendorffii in Gouty Arthritis: Antihyperuricemic, Anti-Inflammatory, and Xanthine Oxidase Inhibition"
}

\author{
Ping Zhao, ${ }^{1}$ Ke-li Chen, ${ }^{1}$ Guo-li Zhang, ${ }^{1,2}$ Guang-rui Deng $\mathbb{D}$, ${ }^{1,2}$ and Juan Li $\mathbb{D}^{1,3}$ \\ ${ }^{1}$ Key Laboratory of Ministry of Education on Traditional Chinese Medicine Resource and Compound Prescription and \\ College of Pharmacy, Hubei University of Chinese Medicine, Wuhan, Hubei 430065, China \\ ${ }^{2}$ Department of Pharmacy, Huanggang Hospital of Traditional Chinese Medicine, Huanggang 438000, China \\ ${ }^{3}$ State Key Laboratory of Dao-Di Herbs, National Resource Center for Chinese Materia Medica, \\ China Academy of Chinese Medical Sciences, Beijing, China
}

Correspondence should be addressed to Guang-rui Deng; ruiqilin@126.com and Juan Li; 1z198207@126.com

Received 13 May 2018; Accepted 28 May 2018; Published 5 July 2018

Copyright (C) 2018 Ping Zhao et al. This is an open access article distributed under the Creative Commons Attribution License, which permits unrestricted use, distribution, and reproduction in any medium, provided the original work is properly cited.

In the article titled "Pharmacological Basis for Use of Selaginella moellendorffii in Gouty Arthritis: Antihyperuricemic, Anti-Inflammatory, and Xanthine Oxidase Inhibition" [1], there was a missing grant number. Accordingly, the Acknowledgments section should be corrected as follows:

This work was funded by China Postdoctoral Science Foundation (2016M601237), the Natural Science Foundation Project (30470193), the Scientific Research Foundation Project of the Education Department of Hubei Province (Q20142007), Natural Science Foundation of Hubei Province (2013CFC055), and the Young Plan Project of Hubei University of Chinese Medicine (XJ2014KJ008).

\section{References}

[1] P. Zhao, K. Chen, G. Zhang, G. Deng, and J. Li, "Pharmacological basis for use of Selaginella moellendorffi in gouty arthritis: antihyperuricemic, anti-inflammatory, and xanthine oxidase inhibition," Evidence-Based Complementary and Alternative Medicine, vol. 2017, 10 pages, 2017. 


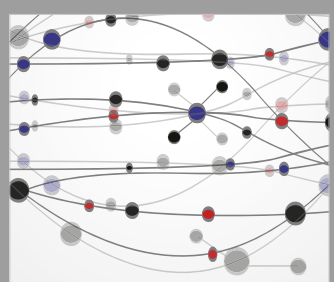

The Scientific World Journal
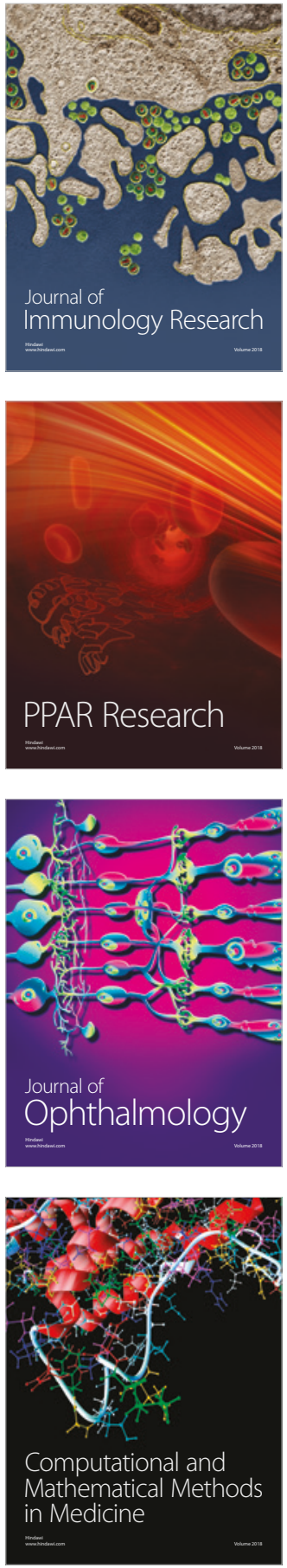

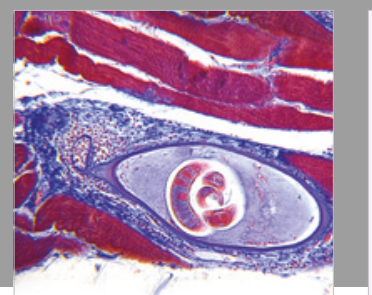

Gastroenterology Research and Practice

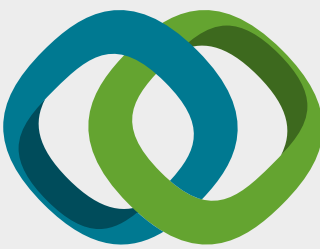

\section{Hindawi}

Submit your manuscripts at

www.hindawi.com
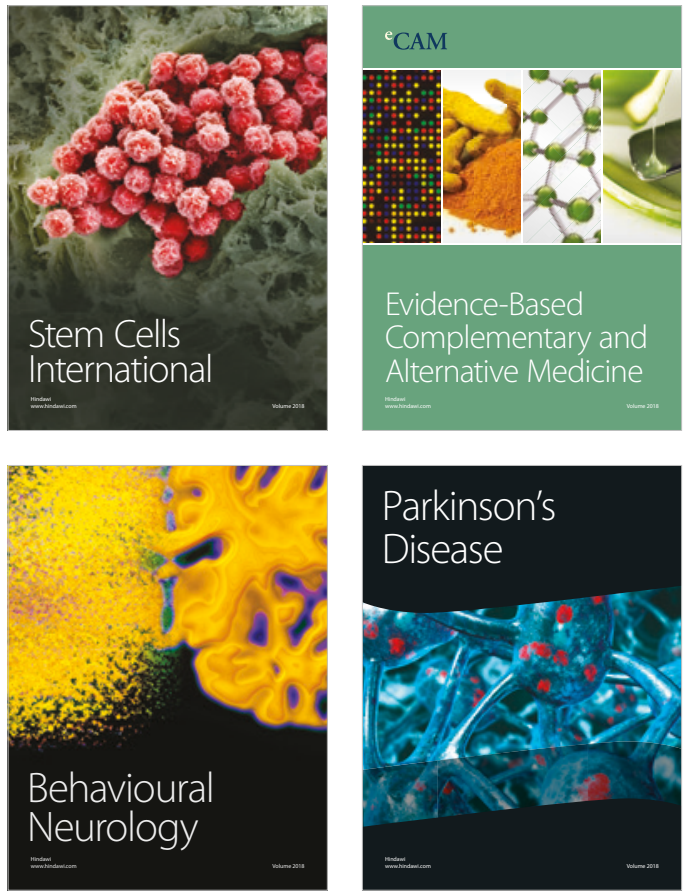

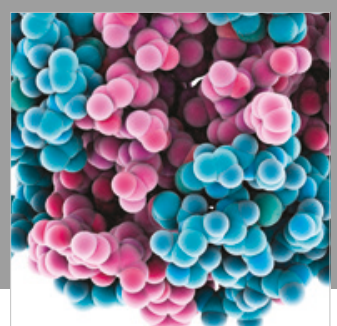

ournal of

Diabetes Research

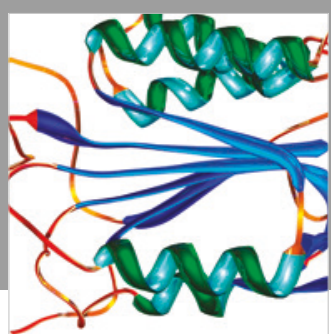

Disease Markers
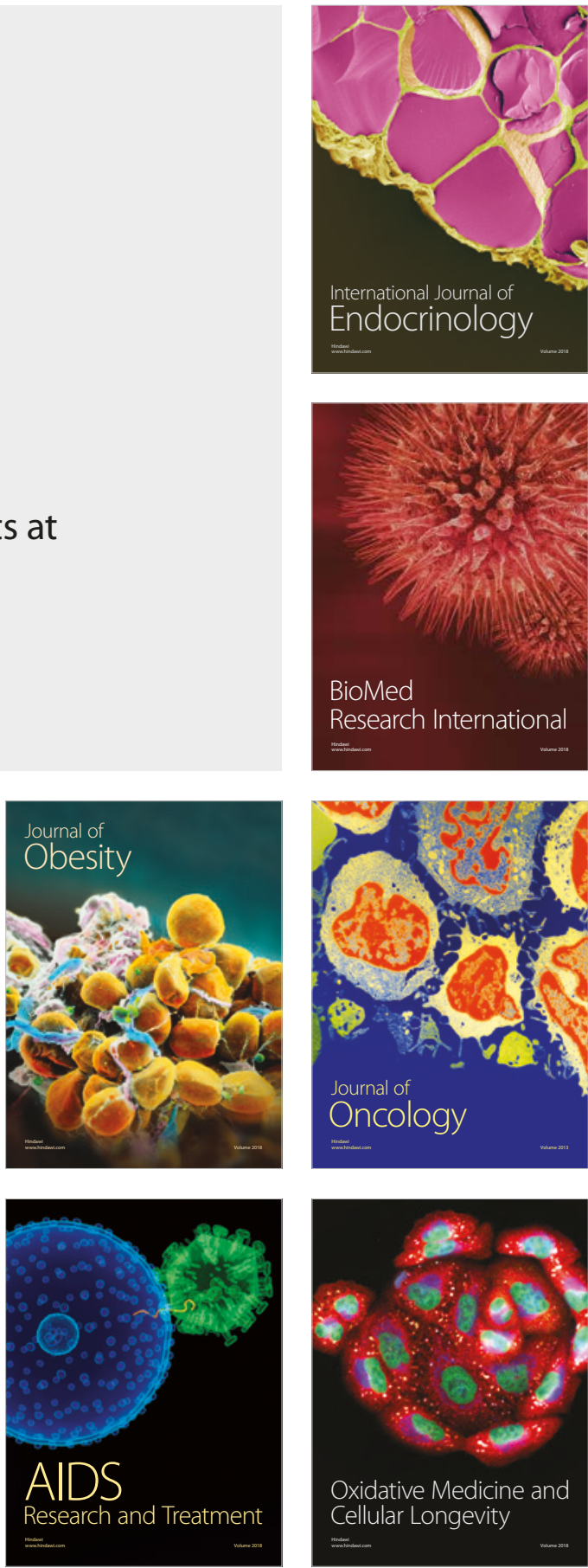